\title{
Speed analysis of the radial tire on hydroplaning pavement
}

\section{CHENG Gang ${ }^{1, a^{*}}$, ZHANG Shihao ${ }^{1, b}$, ZHAO Guoqun $^{2}$, CHENG Qiang ${ }^{3}$, WANG Zhonglei ${ }^{1}$ and LIU $\mathrm{Na}^{1}$}

\author{
${ }^{1}$ School of mechanical and electronic engineering, Key Laboratory of Mechanical Engineering \& \\ Innovation Technology, Shandong Jianzhu University, Jinan, China \\ ${ }^{2}$ Engineering Research Center fo r Mould \& Die, Shandong University, Jinan, China \\ ${ }^{3}$ School of Mechanical and Automotive engineering, Qilu University of Technology, Jinan, China \\ achenggang@sdjzu.edu.cn, b1464844544@qq.com
}

Keywords: Radial tire; Wet pavement; Hydroplaning; Critical velocity.

Abstract. When the vehicle is running on the wet road, the contact force between tire and road decreases and the driving controllability of the vehicle becomes worse due to the hydrodynamic force of water. The finite element model of the steel belted radial tire hydroplaning with complex pattern was derived in the paper. As for the tire part, the Lagrangian method was utilized to simulate the tire motion and deformation. The whole tire meshes was constructed through dividing the one into the pattern and tire body and then cluing together with tie function. Through the simulation of the tire running process on the dry/wet pavement, the Variation of the contact force between the tire and the ground are compared in these two cases with the increased velocity. Meanwhile application of the Momentum theorem, the hydroplaning velocity formula is established. And the relationship between the critical velocity and contact force is obtained.

\section{Introduction}

It is hard to avoid driving on pavement with water or snow mixture, etc. where hydroplaning may occur to automobile tires at high speeds given the extensive application of automobiles as the important role of traffic tools in modern society. When hydroplaning occurs to tires, friction between tire surface and pavement will be significantly reduced. Especially when certain speed limit is exceeded the tire may lose its drive force and braking force completely due to the dynamic pressure of water layer, which may result in immediate threats to personal safeties of passengers and drivers. Therefore, it is an important part of performance analysis of automobile running to analyze the speed of it on hydroplaning pavement.

Not only the complex material structures of tire but also fluid characteristics as well as interaction problems between tire structure and fluid and dynamic characteristics of tire are to be taken into account for simulation analysis of hydroplaning, hence providing certain difficulty to study in such respect. As a result, the simulation study with regard to it did not start until 1980s. Grogger et al[1] conducted some preliminary studies in respect of numerical simulation of tire hydroplaning that it was interpreted mathematically by multi-purpose fluid dynamics suite and internal structural analysis law; however, the tire model was limited to static skidding tire and tire with circumferential pattern. Some experimental studies were carried out, photos through a glass plate were taken to provide visual images of a rolling tire contact shape in water [2]. However, these experimental approaches need tire manufacturing and a test set-up, which entails a comparatively long time and large amount of money. Jenq and Chiu[3] used LS-DYNA software to simulate hydroplaning of a tire with 
consideration of the viscous effect. Okano et al[4] incorporated the rotational speed of tire into simulated analysis of tire hydroplaning characteristics and processed the complex fluid-structure interaction with the help of finite element software that predicted key factors casing tire slip, such as critical speed of hydroplaning, etc. Seta et al.[5] verified the predictability of the hydroplaning simulation in the different parameters such as the water flow, the velocity dependence of hydroplaning, and the effect of the tread pattern on hydroplaning. Choi et al. [6] investigated the parametric characteristics of the snow traction to the snow hardness, the block depth and the tread pattern by MSC/Dytran. The interaction between the tire tread blocks and the snow deformation is implemented by the explicit Euler-Lagrangian coupling scheme. Wies B. et al. [7] studied total void, longitudinal, and lateral void distributions of basic winter, summer tread patterns and tread compounds based on the DOE. Cho et al. [8] estimated a wet-road braking distance for the vehicles equipped with $\mathrm{ABS}$. The operation of $\mathrm{ABS}$ was numerically implemented by controlling the tire angular velocity. Jenq et al.[9] studied the transient hydroplaning behavior of $200 \mathrm{kPa}$ inflated pneumatic radial tires with various types of tread patterns, and obtained dynamic normal contact force and hydroplaning velocity of tire with a prescribed smooth tread pattern. Kim and Jeong [10] developed a new methodology using FDM (Finite Difference Method) code and an FE tire model to calculate the hydroplaning speeds of patterned tires.

A 3D finite element model of radial tire will be established in this paper to simulate the process of circumferentially patterned tire running on the pavement with water flow of certain thickness and analyze the change of contract force between tire and pavement along with increase of speed on dry road and wet road (with water flow). In the meanwhile, to discuss the relations between tire load and the critical speed of hydroplaning by applying the theorem of momentum from the standpoint of tire dynamic hydroplaning mechanism.

\section{Simulation of tire hydroplaning}

When the tire is running on ground with certain amount of water flow, the interaction between it and the pavement has three action fields which are water flow, transition and contact fields [11]. Owing to the complex structure of tire and material nonlinearity, tire modeling takes great efforts that give rise to numerous inconveniences to tire structure and performance analyses. To achieve high-efficient and automatic finite element modeling and analysis of tire, the finite element model parametric study of tire includes not only numerical parameterization of structure dimension geometrically but also some other parameterization processing measures such as boundary conditions and loads, etc.

\section{Tire modeling}

Tire has complex structure that it needs to be reasonably simplified in the course of finite element modeling to acquire a simplified tire structure to the maximum extent under the condition of maintaining simulation precision by ignoring some fine structures not affecting analysis and combining parts made of same materials. The rim can be reduced to a rigid and thin shell element. Cord fabric, belted layer and carcass are composite materials. Calculate basic calculation parameters based on the empirical formula of classic mesomechanics by referring to the structural theories of composite materials. In the meantime, the issue of element mesh generation also needs to consider during modeling. The compacter the element mesh generation, the more time it takes to computing for a CPU, the better the quality of an element and the matrix tends to convergence more. Therefore, factors of all respects shall be taken into account for tire modeling. All tire parts of other materials use continuous solid element representation other than the rim using shell and thin element. 
The pattern on tire thread imposes great effects on tire hydroplaning. Patterned tire and other parts of tire will have their own modelling in this paper and the both models will be combined by rigid connection to eliminate coincident points of interface while simultaneously keep a tight junction of pattern and tread.

\section{Water flow modeling}

It is important to choose water flow modeling with caution in the process of simulation of tire hydroplaning. Considering the viscosity of water flow and limitation to use software, the water flow uses an 8-node solid element representation with thickness of $12 \mathrm{~mm}$. It is the solid ground under of rigid parameter to ensure the control on degree of freedom downward. At the same time, a thick air layer is defined above the water flow facilitating water scattering when thread pattern drains away water. To ensure the fluid-structure interaction between thread pattern and water flow lessen calculations to the largest extent, detailing treatment can be given to the mesh of contact field of water while other parts can use larger meshes.

\section{Load and boundary condition processing}

The fluid-structure interaction method used in this paper can represent the complex interaction of tire deformation and surrounding fluids properly and the tire deformation and fluid analysis will be analyzed respectively using Finite Element Method (FEM) and Finite Volume Method (FVM). In practical applications as shown in the figure below, tire modeling uses the Lagrange body while the fluid modeling uses the Euler body and the interaction of tire deformation and fluid is processed with general interaction algorithm.

In this study, a pressure of $4000 \mathrm{~N}$ downward is imposed to tire and the inflation pressure is one atmospheric pressure to simulate actions of vehicle weight and load on tire. The rolling of tire model on the ground is defined as a rotation motion circling tire axis. There is friction contact between ground and tire and the static and dynamic friction coefficients are 0.3 and 0.5 respectively.

\section{Analysis of simulation result}

By the analogue simulation of tire running on dry pavement and wet water pavement (with water flow) the relations between the contact force and speed can be obtained as shown in the figure below. The contact force between tire and ground on dry pavement which basically keeps unchanged mainly includes the air pressure inside of tire and reactive force of loading. However, when the automobile is running on a road with water flow the contact force will be significantly reduced. The major reason is attributed to the dynamic fluid pressure between tire and water flow. The horizontal component of acting force of water flow acts on the negative direction of running that makes automobile running resistance increase and the vertical component neutralizes loading force of tire lifting the tire. When the acting force of water flow exceeds vertical acting force of tire per se, the tire will separate from the flow completely causing the dangerous condition of complete hydroplaning.

\section{The relation between critical hydroplaning speed and load}

It can be seen from above that the larger the running speed of an automobile, the smaller the contact force between tire and ground and the easier to cause the dangerous condition of hydroplaning. The theory of hydromechanics is used in this paper to verify the relative relation between critical hydroplaning speed and load borne by tire.

Assuming a tire is under fixed load and the pressure borne where tire contacts with ground is uniform and equivalent to loading pressure. Viscous effect of water is ignored that water is considered as an incompressible fluid. The center axis of model is the axis of the tire. In order to facilitate calculations, tire running on water flow is represented by using water flow of certain quantity to impact a tire at a high speed. 


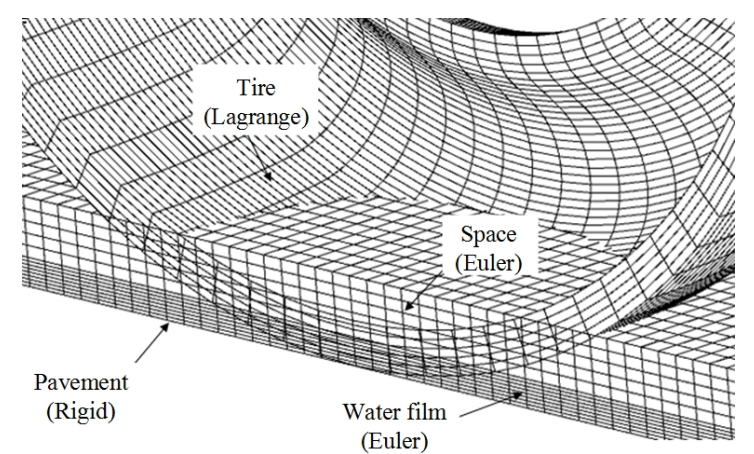

Fig. 1 The model of the tire and water film

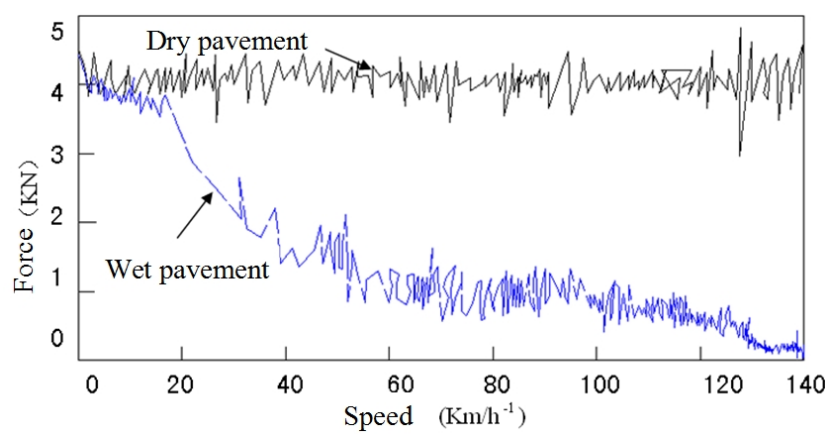

Fig. 2 The diagram of the tire between speed and contact force on dry, wet pavement

When the water flow is thicker, the contact area between water flow and tire is larger. Where analysis the force of the interaction of water flow and tire in unit time (see Figure 5), take the differential section $\mathrm{d}^{\theta}$ and by the theorem of momentum along horizontal $\mathrm{x}$ direction:

$$
d F_{x}=\rho B V^{2} R \sin \theta d \theta
$$

Vertical y direction:

$$
d F_{y}=d F_{x} \cot \theta=\rho B V^{2} R \cos \theta d \theta
$$

By intergration of vertical y direction:

$$
\begin{aligned}
& \mathrm{F}^{\mathrm{y}}=\int_{0}^{\arccos (R-H) / R} \rho B V^{2} R \cos \theta d \theta \\
= & \rho B V^{2} R \sqrt{1-(R-H)^{2} / R^{2}} \\
= & \rho B V^{2} \sqrt{2 H R-H^{2}} \quad(H<R)
\end{aligned}
$$

When $F_{y}$ equals to the inflation pressure of tire loading $G$, the tire is in critical hydroplaning condition, and substitute to above equation to obtain:

$$
V^{2}=G / \rho B \sqrt{2 R H-H^{2}} \quad H<R
$$

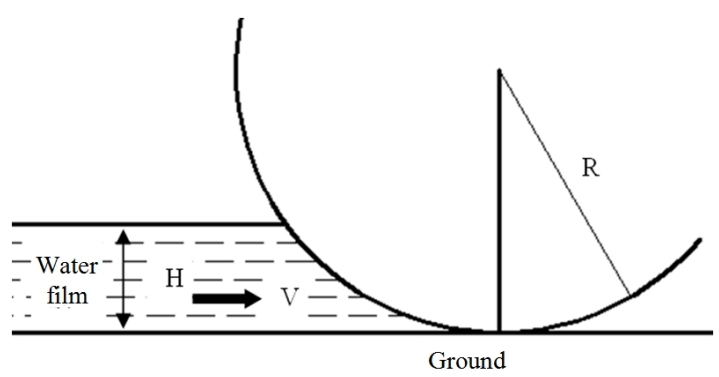

Fig.3 The critical state of the tire hydroplaning

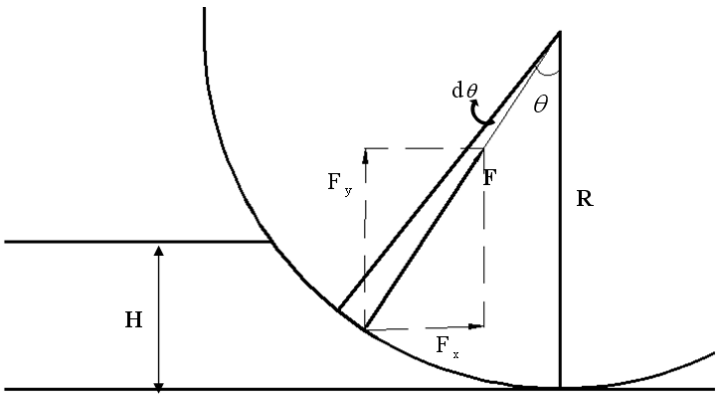

Fig.4 The diagram of the tread stress analysis in hydroplaning critical state

It can be shown from the final result that under certain thickness of water flow the critical speed of tire hydroplaning is increasing along with the increase of its load and inflation pressure within permissible range of tire load-bearing. 


\section{Summary}

The tire-water surface-pavement finite element modeling is achieved by simulation of patterned tire running on wet pavement. It is learnt from force analysis that the cause of tire hydroplaning is the vertical acting force being larger than downward pressure of tire per se. Changing curves of the contact force between tire and pavement on dry and wed road at different speeds are obtained from simulation results. From those curves, it can be seen that the extreme insecurity of tire running on wet and slippery pavement at high speeds. In the meanwhile, the critical speed of tire running on wet and slippery pavement applying the theorem of momentum is $V^{2}=G / \rho B \sqrt{2 R H-H^{2}}$, which indicates that the larger the load of an automobile within in certain limit, the larger of critical hydroplaning speed of tire.

\section{References}

[1] H. Grogger, M. Weiss, Calculation of the three dimensional free surface flow around and automobile tire, Tire Science and Technology. 24(1996) 39-49.

[2] T. Suzuki, T. Fujikawa, Improvement of hydroplaning performance based on water flow around tires, SAE World Congress. (2001)

[3] S.T. Jenq, Y.S. Chiu, Hydroplaning analysis for tire rolling over water film with various thicknesses using the LS-DYNA fluid-structure interactive scheme, Computers, Materials and Continua. 11(2009) 33-58 .

[4] T. Okano, M. Koishi, A new computational procedure to predict transient hydroplaning performance of a tire, Tire Science and Technology. 29 (2001) 2-22.

[5] E. Seta , Y. Nakajima, T. Kamegawa, H. Ogawa, Hydroplaning analysis by FEM and FVM: Effect of tire rolling and tire pattern on hydroplaning, Tire Science and Technology. 28(2000) 140-156.

[6] J. H. Choi, J.R. Cho, J.S. Woo, K.W. Kim, Numerical investigation of snow traction characteristics of 3-D patterned tire, Journal of Terramechanics. 49(2012) 81-93.

[7] B. Wies, B. Roeger, R. Mundl, Influence of Pattern Void on Hydroplaning and Related Target Conflicts, Tire Science and Technology. 37(2009) 187-206.

[8] J. R. Cho, H. W. Lee, W. S. Yoo, A wet-road braking distance estimate utilizing the hydroplaning analysis of patterned tire, International journal for numerical methods in engineering. 69(2007) 1423-1445.

[9] S. T. Jenq, Y. S. Chiu, Hydroplaning Analysis for Tire Rolling over Water Film with Various Thicknesses Using the LS-DYNA Fluid-Structure Interactive Scheme, Computers, Materials \& Continua. 11(2009) 33-58

[10] T. W. Kim, H. Y. Jeong, Hydroplaning simulations for tires using FEM, FVM and an asymptotic method, International Journal of Automotive Technology. 11(2010) 901-908. [11] S.K. Agrawall , J.J.Henry, A simple tire deformation model for the transient aspect for hydroplaning, Tire science and technology. 8 (1980) 23-35. 